\begin{tabular}{|c|c|}
\hline Title & A rterioportal shunting as an alternative to microvascular reconstruction after hepatic artery resection. \\
\hline Author(s) & Kondo, S.; Hirano, S.; A mbo, Y.; Tanaka, E.; Kubota, T.; Katoh, H. \\
\hline Citation & $\begin{array}{l}\text { British Journal of Surgery, 91(2), 248-251 } \\
\text { https://doi.org/10.1002/bjs.4428 }\end{array}$ \\
\hline Issue Date & 200402 \\
\hline Doc URL & http:/hdl.handle.net/2115/15851 \\
\hline Rights & Copyright @ 2004 John Wiley \& Sons, Inc., British Journal of Surgery, V ol. 91-2, pp. $248-251$ \\
\hline Type & article (author version) \\
\hline File Information & BJS91-2.pdf \\
\hline
\end{tabular}

Instructions for use 


\section{Arterioportal shunting as an alternative to microvascular reconstruction after hepatic artery resection}

Satoshi Kondo, MD¹; Satoshi Hirano, MD ${ }^{1}$; Yoshiyasu Ambo, MD ${ }^{1}$; Eiichi Tanaka, MD ${ }^{1}$; Takehiro Kubota, MD²; and Hiroyuki Katoh, MD¹

Departments of Surgical Oncology ${ }^{1}$ and Cardiovascular Surgery ${ }^{2}$

Hokkaido University Graduate School of Medicine

N15 W7, Kita-ku, Sapporo 060-8638, Japan

Corresponding author and reprint request:

Satoshi Kondo, MD

Department of Surgical Oncology, Division of Cancer Medicine

Hokkaido University Graduate School of Medicine

N15 W7, Kita-ku, Sapporo 060-8638, Japan

Phone: +81-11-706-7714

Fax: +81-11-706-7158

Email: kondows@med.hokudai.ac.jp 
Background: Portal vein and hepatic artery resection and reconstruction may be required in radical surgery for biliary cancer. Microvascular reconstruction requires special equipment and training, and may be difficult to accomplish when the arterial stump is small, there are multiple vessels or it lies deep within the wound. This study examined the feasibility and safety of arterioportal shunting as an alternative to arterial reconstruction. Methods: Over 30 months, ten patients with biliary cancer (bile duct carcinoma in six and gallbladder carcinoma in four) underwent radical surgery with en bloc resection of the hepatic artery and end-to-side arterioportal reconstruction between the common hepatic or gastroduodenal artery and the portal trunk.

Results: No patient died. Complications included bile leakage in two patients and liver abscess in one. Routine angiography performed 1 month after surgery revealed shunt occlusion in three patients. Once the existence of hepatopetal arterial collaterals had been confirmed in the remaining patients, the shunt was occluded by coil embolization.

Conclusion: Arterioportal shunting represents a safe alternative to microvascular reconstruction after hepatic artery resection. However, its potential to increase the rate of cure and safety require further assessment in a larger series with longer follow-up. 


\section{Introduction}

_L_ Locally advanced biliary cancer often involves the portal vein and hepatic artery within the hepatoduodenal ligament, and concomitant resection may be required for cure. Perineural invasion, a common mode of spread in biliary cancer ${ }^{1,2}$, may necessitate concomitant hepatic artery resection even when there is no cancer cell infiltration into the arterial wall because the autonomic nerve plexus surrounds the hepatic artery circumferentially Portal vein resection is now considered a safe procedure with favourable results ${ }^{3-6}$. However, hepatic artery resection is less common, because the reconstruction is more complicated and requires microvascular techniques ${ }^{7,8}$. The anastomosis may be difficult or impossible to perform when the arterial stump is small, there are multiple vessels, or it is located deep within the wound.

\section{An experimental study using dogs subjected to hepatobiliary}

dearterialization demonstrated that arterioportal shunting (APS) reduced acute hypoxic hepatic injury and improved hypoxia in the bile duct, permitting successful choledochojejunostomy ${ }^{9,10}$. There are however, few reports of the clinical application of APS and its clinical consequences are as an alternative to microvascular anastomosis after hepatic artery resection in radical surgery for biliary cancer. 


\section{Patients and methods}

Over a 30 month period from May 2000, ten patients with biliary cancer (bile duct carcinoma in six and gallbladder carcinoma in four) have undergone radical surgery with en bloc resection of the hepatic artery and

APS. The first patient had undergone left hepatectomy and APS was employed at an emergency operation to salvage a dearterialized hepatobiliary system that resulted from accidental injury to a right hepatic artery encased by tumour. Because the distal stumps of the right hepatic artery were small, multiple and deeply located, microvascular anastomosis was abandoned in favour of APS. In the remaining nine patients, preoperative investigation with computed tomography and intraductal ultrasonography suggested involvement of the hepatic artery and informed consent was obtained before treatment.

All patients were men, aged between 56 and 81 (median 69) years. Hilar bile duct resection involving division of the left, right anterior, right posterior and caudate ducts individually, was performed in all patients and was used alone in four patients. One patient required a local wedge excision of adjacent liver, Left hepatectomy was performed for radical resection of the tumour in two patients and this was extended to the right anterior segments in a further patient. Two patients required concomitant pancreaticoduodenectomy, one of whom also needed a wedge hepatectomy. These Three patients underwent portal vein resections. The concomitantly
削除: [Author: Table 1 has been deleted because it gives details for individual patients. Most of the data in the table were in the original text anyway. However, there were some discrepancies, as noted below. Please ensure that correct figures are given in final text]During the

削除: $\mathrm{s}$

削除: since

削除: underwent

削除:

削除:

削除:

削除: , defined as

削除:

削除: L

削除: -sided major

削除: hree

削除: pancreatoduodenectomy in two patients; otherwise, hilar bile duct resection alone was

performed[According to Table 1, only four patients had hilar bile duct resection alone, cf. $10-5=5$ here]. Wedge resection of the portal vein and partial duodenectomy were required in three and two patients respectively[Author: Wedge resection of the portal vein in three patients and partial duodenectomy in two are not noted in Table 1; on the other hand the table notes wedge hepatectomy in two patients].

書式変更：蛍光ペン 
resected hepatic arteries were the proper and right hepatic arteries in the three patients who underwent left-sided major hepatectomy and the right hepatic artery alone with preservation of the left hepatic artery in six patients. The final patient had marked perineural invasion in the plexus surrounding the hepatic artery, necessitating resection of the entire hepatic artery including the distal part of the common hepatic artery.

\section{Surgical technique}

After removing the specimen and before biliary reconstruction, an arterioportal shunt was created in an end-to-side fashion between the common hepatic artery (when both the right and left hepatic arteries were resected) or the gastroduodenal artery (when the left hepatic artery was preserved) and the portal trunk. Side clamping of the portal vein allowed the vein to remain patent during the vascular anastomosis. Interrupted 9/0 polypropylene (Ethicon, Edinburgh, UK) sutures were placed with the aid of

削除: Prolene[Author: Is Prolene ${ }^{\circledR}$ an operating microscope in the first two patients; thereafter a continuous polypropylene $6 / 0$ suture was employed using loupes. A thrill was palpable on the wall of the portal vein after successful creation of the shunt or TM?] 


\section{Results}

\section{Perioperative results}

The operating time ranged from 538 to 896 (median 759) min. The operative blood loss varied between 515 and 2880 (median 1720) $\mathrm{ml}$ and three patients required red blood transfusion. The serum concentrations of aspartate aminotransferase and alanine aminotransferase one day after operation ranged from 92 to 1265 (median 180) and 87 to 785 (median 125) units/1 respectively both returning to normal within a few days. The maximum postoperative concentration of total serum bilirubin was $22-71$ (median 42$) \underline{\mu m o l} / 1 . \quad$ No patient developed hepatic failure.

There were no deaths, although three patients suffered complications.

Bile leakage developed in two patients, from the hepaticojejunostomy in one and of unknown origin in the other. The leakage resolved with external drainage within 2 weeks.

Angiography was performed 4 to 5 weeks after surgery in all patients.

The arterioportal shunt was found to be occluded in three patients and hepatopetal arterial collaterals had developed. In the remaining seven patients, the shunt was occluded by coil embolization to prevent portal hypertension in future, after confirming the presence of a collateral arterial supply to the liver via the hepaticojejunostomy, the inferior phrenic artery or the contralateral hepatic artery. A liver abscess developed immediately after embolization in one patient and was successfully managed by 
percutaneous drainage.

\section{Pathological findings}

__ Histological examination of resected specimens revealed a positive surgical margin in two patients; otherwise, the resections were considered curative. Infiltration of the hepatic artery was detected in two patients and primary tumour and/or perineural invasion close to the arterial wall in the other eight patients. Lymph node metastasis and perineural invasion had occurred in six and seven patients respectively.

\section{Postoperative survival}

Three patients died from their disease 23,21 and 11 months after surgery from local, hepatic and peritoneal recurrences respectively patient died suddenly from an unknown cause at another hospital 1 month after discharge. The remaining six patients are alive $26,11,8,4,3$ and 1 month after surgery.

削除: recurren

削除: The recurrence was 削除: [Author: should 'respectively' be added here, or did all three patients have all types of recurrence?]. 


\section{Discussion}

__ In radical hepatobiliary surgery, the peribiliary arterial network is disrupted by skeletonization. Therefore, total interruption of the hepatic arterial flow may cause serious postoperative complications related to biliary ischaemia, including disruption of the bilioenteric anastomosis and liver abscess, even though hypoxic hepatic parenchymal injury does not occur as long as portal flow is maintained ${ }^{12,15-17}$. Restoration of biliary arterial flow seems important , Arterial reconstruction using a microvascular anastomosis is useful 7,8 , but is difficult or impossible in some patients.

The biliary blood supply depends primarily on hepatic arterial flow, and APS has been devised as a way of improving hepatic function and preventing liver failure ${ }^{10,18}$. An experimental study demonstrated that APS improved biliary oxygen saturation after hepatobiliary dearterialization from 28 per cent of normal to 57 per cent ${ }^{9}$. The mechanism responsible is unclear, although back-flow and/or back-diffusion from the sinusoidal network or presinusoidal shunt has been suggested.

Hepatopetal arterial collaterals developed in all ten patients within one month in the present study and the shunt was closed when still patent to prevent portal hypertension. Clinical cases of portal hypertension and oesophageal varices after APS have been reported ${ }^{12}$. Whether biliary ischaemia-related complications develop or not seems to depend on the total

削除: Although this improvement maintains the viability of the bile duct and ensures the integrity of the bilioenteric anastomosis, APS is not sufficient as a single, permanent blood supply to the bile duct.

\section{削除: 1}

削除: . It was therefore possible to close

削除: arterioportal

削除: This is more than a theoretical concern as c 
oxygen supply to the bile duct through the arterioportal shunt and the arterial collaterals. Therefore, once the arterial collaterals have been established, the arterioportal shunt is no longer necessary.

APS is simpler than microvascular anastomosis and may be used when arterial reconstruction is impossible. Furthermore, the use of an arterioportal shunt may increase the proportion of patients where surgery might be considered curative when hepatic aterial resection is necessary, The hepatic artery does not need to be isolated within the hepatoduodenal ligament so that en bloc resection of the hepatic artery and the surrounding nerve sheath, potentially harbouring perineural invasion, may increase 削除: cure rate among patients who require hepatic arterial resection.

削除: between the origin of the proper or right hepatic artery and its peripheral branches at level at which the hepatic ducts divide, minimizing the plane of dissection

削除: [Author: Is this sentence OK?]. E curability. This is particularly true in cases of resection of the right hepatic artery, which runs close to the hilar bile duct. Six patients in the present study underwent resection of the right hepatic artery and APS for this reason,

As Miyazaki et al. ${ }^{17}$ and Tsuge et al. ${ }^{15}$ have pointed out, right hepatic artery resection does not require arterial reconstruction as long as the hepatic ductal confluence or the hilar plate ${ }^{19}$ is preserved because interlobar arterial communications are adequate. In the present series, however, all patients underwent resection including the hilar plate, which mandated arterial reconstruction or APS.

Iseki et al. ${ }^{12}$ reported three successful cases of concomitant hepatic artery resection with APS for pancreatobiliary cancer and stated that

削除: the

\section{削除: is}

削除: hilar bile duct 
mesenteric APS has the advantages of choice of arterial size, a site removed from the primary operative field and ease of shunt closure by transarterial embolization. The authors employ hepatic or gastroduodenal APS rather than mesenteric APS based on experimental evidence that shunt flow similar to original hepatic arterial flow $_{2}$ is optimal ${ }^{9,10}$.

The satisfactory perioperative results in the present study endorse the feasibility and safety of APS. The effect of APS in three patients whose shunt was occluded at the time of postoperative angiography could not be assessed because the duration of patency was unknown. The incidence of bile leakage js similar to the incidence seen after radical surgery without hepatic artery resection and did not require further surgical intervention, ,The late development of a liver abscess in one patient was temporally related to coil embolization of the shunt, although the pathogenesis was obscure.

APS appears to be a safe alternative to microvascular reconstruction after hepatic artery resection in radical surgery for biliary cancer. The safety of the procedure and its potential for increasing curability, need to be confirmed in a larger series of patients.

削除: (jejunal or ileal)

削除:

削除: The present experience, however, has shown that these considerations are not problematic in hepatic or gastroduodenal APS.

削除: the

削除: the

削除: In fact, two of the three patients in Iseki's series developed liver abscess or anastomotic leakage, and all three patients had

postoperative hyperbilirubinaemia. The incidence of these complications was higher than in the present study. This difference may be attributable to less flow in a mesenteric

arterioportal shunt. Successful concomitant hepatic artery resection with gastroduodenal APS for biliary cancer, with no postoperative complications, has been reported previously ${ }^{14}$.

削除: (two patients)

削除: wa

削除: of

削除: [Author: Is this sentence correct?]

削除: Delayed occurrence of

削除: However, greater attention should be paid to the fact that two of the three patients who underwent major hepatectomy developed a postoperative complication. This implies that oxygen supply to the bile duct through the shunt alone may be insufficient.

\section{削除:}

削除: was shown to be

削除: However, $\mathrm{t}$

削除: improving the cure rate

削除: with long-term follow-up 


\section{References}

1. Bhuiya MR, Nimura Y, Kamiya J, Kondo S, Fukata S, Hayakawa N, et al. Clinicopathologic studies on perineural invasion of bile duct carcinoma. Ann Surg 1992; 215:344-349.

2. Yamaguchi R, Nagino M, Oda K, Kamiya J, Uesaka K, Nimura Y. Perineural invasion has a negative impact on survival of patients with gallbladder carcinoma. Br J Surg 2002; 89:1130-1136.

3. Neuhaus P, Jonas S, Bechstein WO, Lohmann R, Radke C, Kling N, et al. Extended resections for hilar cholangiocarcinoma. Ann Surg 1999; 230:808-819.

4. Nimura Y, Kamiya J, Kondo S, Nagino M, Uesaka K, Oda K, et al. Aggressive preoperative management and extended surgery for hilar cholangiocarcinoma: Nagoya experience. J Hepatobiliary Pancreat Surg 2000; 7:155-162.

5. Munoz L, Roayaie S, Maman D, Fishbein T, Sheiner P, Emre S, et al. Hilar cholangiocarcinoma involving the portal vein bifurcation: long-term results after resection. J Hepatobiliary Pancreat Surg 2002; 9:237-241.

6. Kondo S, Katoh H, Hirano S, Ambo Y, Tanaka E, Okushiba S. Portal vein resection and reconstruction prior to hepatic dissection during right hepatectomy and caudate lobectomy for hepatobiliary cancer. Br J Surg 2003; 90:694-697. 
7. Yamamoto Y, Sugihara T, Sasaki S, Furukawa H, Furukawa H, Okushiba S, et al. Microsurgical reconstruction of the hepatic and superior mesenteric arteries using a back wall technique. J Reconstr Microsurg 1999; 15:321-325.

8. Yamanaka N, Yasui C, Yamanaka J, Ando T, Kuroda N, Maeda S, et al. Left hemihepatectomy with microsurgical reconstruction of the right-sided hepatic vasculature: a strategy for preserving hepatic function in patients with proximal bile duct cancer. Langenbecks Arch Surg 2001; 386:364-368.

9. Munemura T. An experimental study of blood supply to the bile duct under arterio-portal shunting (in Japanese with English abstract). Hokkaido J Med Sci 1995; 70:591-608.

10. Suzuki O, Takahashi T, Kitagami H, Manase H, Watanabe S, Kondo S, et al. Appropriate blood flow for arterio-portal shunt in acute hypoxic liver failure. Eur Surg Res 1999; 31:324-332.

11. Iseki J, Touyama K, Noie T, Nakagami K, Takagi M, Hakamada K, et al. Partial portal arterialization for the prevention of massive liver necrosis following extended pancreatobiliary surgery: experience of two cases. Surg Today 1992; 22:568-571.

12. Iseki J, Noie T, Touyama K, Nakagami K, Takagi M, Ori T, et al. Mesenteric arterioportal shunt after hepatic artery interruption. Surgery $1998 ; 123: 58-66$. 
13. Tanabe G, Kawaida K, Hamanoue M, Kihara K, Hirata S, Maemura S, et al. Treatment for accidental occlusion of the hepatic artery after hepatic resection: report of two cases. Surg Today 1999; 29:268-272.

14. Inoue T, Sawa T, Okada S, Kinoshita K, Yoshimitsu S. Partial portal arterialization in complete en bloc resection of the hepatoduodenal ligament and left lobe of the liver for hepatic hilar cancer. Hepatogastroenterology 2000; 47:533-536.

15. Tsuge H, Mimura H, Hamazaki K, Mori M, Kawata N, Orita K. Interruption of hepatic arterial blood flow after resection of pancreaticobiliary carcinoma. Hepatogastroenterology 1995; 42:966-974.

16. Gupta N, Solomon H, Fairchild R, Kaminski DL. Management and outcome of patients with combined bile duct and hepatic artery injuries. Arch Surg 1998; 133:176-181.

17. Miyazaki M, Ito H, Nakagawa K, Ambiru S, Shimizu H, Yoshidome H, et al. Unilateral hepatic artery reconstruction is unnecessary in biliary tract carcinomas involving lobar hepatic artery: implications of interlobar hepatic artery and its preservation. Hepatogastroenterology $2000 ; 47: 1526-1530$.

18. Shimizu Y, Miyazaki M, Shimizu H, Ito H, Nakagawa K, Ambiru S, et al. Beneficial effects of arterialization of the portal vein on extended hepatectomy. Br J Surg 2000; 87:784-789. 
19. Kawarada Y, Das BC, Taoka H. Anatomy of the hepatic hilar area: the plate system. J Hepatobiliary Pancreat Surg 2000; 7:580-586. 